\title{
La avifauna del Recinto Universitario de Paraíso, Cartago, Costa Rica
}

\author{
The avian fauna from the Paraíso University Campus, Cartago, Costa Rica
}

\author{
Víctor J. Acosta-Chaves ${ }^{1}$ \\ Diego Ramírez-Calvo ${ }^{2}$
}

Fecha de recepción: 1-04-2019

Fecha de aprobación: 3-02-2020

\begin{abstract}
Resumen
La pérdida de hábitat ha puesto en peligro la avifauna urbana del Gran Área Metropolitana (GAM) de Costa Rica, aunque campus universitarios y parques urbanos sirven de reservorio para cientos de especies. Presentamos una lista preliminar de las aves del Recinto de Paraíso de la Universidad de Costa Rica (UCR), el cual generamos mediante censo por búsqueda intensiva y aplicación de ciencia ciudadana (2015-2018). Detectamos 120 especies (17 órdenes, 37 familias, 94 géneros) y un híbrido en el campus. Comparamos nuestro listado con otros de campus de la UCR y zonas urbanas de la GAM mediante un análisis de conglomerados (Índice de Jaccard, vinculación simple). El Recinto de Paraíso presentó más de la mitad de la riqueza registrada para la Sede Central (UCR), y más especies que la Sede Occidente (UCR) y demás sitios comparados. La similitud entre la riqueza de aves del Recinto de Paraíso y los demás sitios fue de al menos un 40\%. La ciudad de Paraíso presenta condiciones biogeográficas y de paisaje propicias para una rica avifauna, pero algunos de sus ecosistemas están comprometidos. Por lo tanto, la Universidad de Costa Rica debe conservar sus humedales y fragmentos de bosque, e implementar diseño verde, para proteger la diversidad de aves en sus campus. Palabras Claves: Aves urbanas, biodiversidad urbana, campus universitario, Ciudad de Paraíso, Gran Área Metropolitana, riqueza de aves.
\end{abstract}

\begin{abstract}
The habitat loss has threatened the urban avian fauna of the Great Metropolitan Area (GMA) of Costa Rica, although university campuses and urban parks serve as a reservoir for hundreds of species. We present a preliminary list of the birds of the Recinto de Paraíso Campus of the University of Costa Rica (UCR), which we generated through census by intensive search and application of citizen science (2015-2018). We detected 120 species (17 orders, 37 families, 94 genera) and a hybrid on campus. We compared our list against others on the UCR campus and urban areas of the GMA through a cluster analysis (Jaccard Index, simple linkage). The Recinto de Paraíso encompass more than half of the richness registered for the Sede Central UCR, and more species than the Sede de Occidente (UCR) and other sites compared. The similarity between the richness of the Recinto de Paraíso versus the other sites was at least $40 \%$. The city of Paraíso presents biogeographical and landscape conditions conducive to a rich bird fauna, but some of its ecosystems are compromised. The UCR must conserve its wetlands and forest fragments, and implement green design, to protect the diversity of birds on its campuses.
\end{abstract}

Key Words: Bird richness, City of Paraíso, Great Metropolitan Area, university campus, urban birds, urban biodiversity.

\footnotetext{
1 Magister en Biología. Docente-investigador en Carrera de Turismo Ecológico, Recinto de Paraíso, Sede del Atlántico, Universidad de Costa Rica, Costa Rica y el Centro de Programas Internacionales de la Universidad Veritas, Costa Rica. Correo electrónico: victor.acosta@ucr.ac.cr / victor.acosta@veritas.cr

2 Estudiante de Turismo Ecológico, Recinto de Paraíso, Sede del Atlántico, Universidad de Costa Rica, Costa Rica. Correo electrónico: diego. ramirezcalvo@ucr.ac.cr
} 


\section{Introducción}

Costa Rica es uno de los países con mayor riqueza de avifauna en la región Centroamericana y del Caribe, actualmente se registran más de 920 especies de aves en todo su territorio continental y marítimo (Sandoval y Sánchez, 2019). Debido a esta riqueza, las aves son un punto focal de investigación y atracción turística para Costa Rica, con una tendencia a un crecimiento exponencial en las actividades relacionadas con la avifauna, caracterizadas por una alta participación tanto de especialistas como aficionados (Soto, 2016). No obstante, la pérdida de hábitat por desarrollo agrícola y urbano, sumado a otras problemáticas medio ambientales, en espacios urbanos y periurbanos son factores que afectan negativamente a muchas poblaciones de vertebrados como las aves (Guido y Rodríguez, 2009; Biamonte, Sandoval, Chacón y Barrantes, 2011; Acosta, 2013), creando factores de riesgo y vulnerabilidad para este grupo de organismos.

Los procesos de urbanización son importantes modificadores del paisaje pues generan nuevos ambientes y situaciones particulares para las comunidades de aves, a veces incluso favoreciendo a ciertas especies adaptables a la urbanización (Leveau y Leveau, 2004); pero, en la mayoría de las ocasiones, incrementando la tasa local de extinción y pérdida de diversidad (Biamonte et al., 2011). Algunas coberturas vegetales dentro de ciudades son un remanente de los ecosistemas originales consumidos por la urbanización, e incluso estos espacios permiten la subsistencia de una relativamente alta diversidad de aves debido a la heterogeneidad del paisaje (Marín, 2005). En contraste, siendo la diversidad de especies cada vez menor en áreas urbanas (Biamonte et al. 2011; Acosta, 2013), se ha generado un mayor interés por parte de los residentes de áreas urbanas por conocer la biodiversidad presente en sus ambientes cercanos, sobre todo su avifauna (Clergeau, Isaacson, Murton y Falardeau,1998). La investigación de estos espacios abre perspectivas sobre los desafíos y toma de decisiones sobre manejo y conservación en áreas urbanizadas (Clergeau et al., 1998), pues en muchos casos actúan como corredores biológicos o zonas de confinamiento (Marín, 2005; Cerdas-Fernández, 2014).
Antes de los noventas, era poco lo que se conocía sobre avifauna neotropical urbana, las investigaciones y los esfuerzos se enfocaban en crear áreas con menos alteración y mejor preservación (Marín, 2005). No obstante, el desarrollo de estudios con aves en ambientes urbanos se incrementó en los últimos años en América Latina, incluyendo esfuerzos por capturar su diversidad (Escobar y McGregor, 2017). En el Gran Área Metropolitana (GAM) de Costa Rica, la avifauna de ciertos campus y bosques urbanos ha sido registrada históricamente. Por ejemplo, Stiles (1990) detectó 188 especies de aves en el Campus Rodrigo Facio (Sede Central UCR) de la Universidad de Costa Rica (UCR) al Este del GAM desde 1968 hasta 1989. Posteriormente, Biamonte et al. (2011) repitieron dicho esfuerzo en la Sede Central UCR durante los años de 2004 a 2009, pero solo registraron 143 especies, para una lista acumulativa de 207 variedades en dicho campus. Por su parte, Cerdas-Fernández (2014) registró 79 especies de aves en parques del centro de la ciudad de San José durante el año 2010. Mientras que al oeste del GAM (Ciudad de San Ramón), específicamente en el Campus Carlos Monge Alfaro (Sede Occidente UCR), Rodríguez y Guido (2008) registraron 108 especies de aves desde 2006 hasta 2008; además, de 2006 a 2009 documentaron 86 especies en el Bosque Municipal de San Ramón José Figueres Ferrer. Finalmente, Rodríguez y Vargas (2016) detectaron 35 especies para el Campus Omar Dengo de la Universidad Nacional en Heredia, aunque su esfuerzo de muestreo fue bastante menor al de los demás estudios y la riqueza de ese campus podría ser mayor (Acosta, obs. pers.). Muchos de esos estudios sobre avifauna se enfocan en campus universitarios y parques municipales, por ser áreas que mantienen remanentes de vegetación en medio de ambientes altamente urbanizados (Cerdas, 2014).

En este artículo, se presenta el primer listado de las especies de aves que se pueden encontrar en el Recinto de Paraíso, Provincia de Cartago, Costa Rica. Además, comparamos nuestros resultados contra otras listas generadas por estudios durante diferentes periodos en campus y parques del GAM, para calcular el grado de similitud entre los sitios basado en su riqueza. Este esfuerzo permitirá establecer una base para futuras investigaciones sobre la avifauna de la zona. 


\section{Materiales y métodos}

\section{1. Área de estudio}

Se realizó un inventario el Campus Universitario Rafael Ángel Calderón Guardia de la Universidad de Costa Rica, Sede del Atlántico (conocido como Recinto de Paraíso) (949’1”N, 8352'07.0” O, datum: WGS84; 1300 msnm) y sus alrededores del Cantón de Paraíso, Provincia de Cartago, Costa Rica. La zona de vida corresponde al bosque premontano húmedo (Holdridge, 1987), con una precipitación aproximada de $1950 \mathrm{~mm}$ (Molina, Gamboa y González, 1991). El campus cuenta con una extensión de unas cuatro hectáreas, divididas en tres áreas principales: edificaciones, fragmento de bosque reforestado con especies exóticas y nativas (El Bosquecito) y un humedal que cubre un espacio considerable del campus (Figura 1).

El Recinto de Paraíso pertenece a la unidad fitogeográfica Cordillera Volcánica Central (Zamora, 2008). La vegetación característica está dominada por pastizales con especies como el higuerón (Ficus sp.), cortés amarillo (Tabebuia ochracea), lorito (Cojoba arborea), targuá (Croton draco), sotacaballo (Zygia longifolia), Guayaba (Psidium guajava), pino australiano (Casuarina sp.), pino (Pinus sp.) y plantas ornamentales. El humedal pantanoso tipo ciénaga se extiende por aproximadamente 0.735 hectáreas, pero está invadido por lirios acuáticos y pastos de unos $50 \mathrm{~cm}$ de alto que evitan la formación del espejo de agua, aunque mantiene una base húmeda durante el año (Brenes y Brenes, 2016) (Figuras 1-2).

\subsection{Muestreo de aves}

Para registrar la mayor cantidad de especies se aplicó el método de censo por búsqueda intensiva (Ralph et al., 1996). Además de realizar diez muestreos no estandarizados, desde enero de 2016 hasta enero de 2018, recorriendo todo el campus (6-8 h, 15-18h). Además de incluir observaciones esporádicas hechas desde 2015 hasta 2018 en el Curso de Elementos de Biosistemática, así como registros de terceros que aportaron datos debidamente documentados mediante fotografías o grabaciones. Para la identificación visual de las aves se utilizaron las guías de Stiles y Skuch (1989) y Garrigues y Dean (2014), y la base de datos Xeno-canto (Xeno-canto Foundation, 2018) para discriminar ciertas vocalizaciones. Las aves detectadas se clasificaron por orden taxonómico, así como su estatus actual en Costa Rica (R: residente, M: migratorio, R-END: endémico) siguiendo la Lista de Aves de Costa Rica de Sandoval y Sánchez (2019).

\subsection{Análisis de datos}

En primer lugar, se identificó especies que pudieran tener algún grado de amenaza de acuerdo con la lista de especies con poblaciones reducidas y en peligro de extinción para Costa Rica (MINAE, 2005), los apéndices de la Convención de Comercio Internacional de Especies Amenazadas de Fauna y Flora Silvestre (CITES, 2018) y la Lista Roja de Especies Amenazadas (UICN, 2018).

Seguidamente, se comparó el porcentaje de similitud de la avifauna del Recinto de Paraíso con los listados de la Sede Central UCR 1968-1989 (A) y 2004-2009 (B), parques urbanos en San Francisco de Dos Ríos (San José) durante 2010, la Sede Occidente UCR 2006-2008 y el Bosque Municipal de San Ramón 2006-2009 mediante un análisis de conglomerados (vinculación simple, índice de Jaccard) usando el software PAST 3.2. (Hammer, Harper y Ryan, 2001). Finalmente, al comparar dichos listados, se determinó cuáles especies fueron únicas para el Recinto de Paraíso.

\section{Resultados}

Se documentó un total de 120 especies distribuidas en 17 órdenes, 37 familias y 94 géneros (Cuadro 1). Las familias que contribuyeron con un mayor número de especies fueron Tyrannidae, Parulidae y Thraupidae (Figura 3). En términos de estatus de las aves observadas predominaron las residentes (83 especies), seguidas por las migratorias latitudinales (25 especies) (Anexo 1, Figura 4). Este listado, sumado al de la Sede Central y Sede Occidente de la Universidad de Costa Rica, genera un acumulado de 269 especies para los tres campus. 
El Recinto de Paraíso contiene 20 especies de aves que se clasifican dentro de la categoría de Apéndice II de CITES, mientras que Falco peregrino se ubica en el Apéndice I (Anexo 1). Los pericos Pionus senilis, Psittacara finschi, así como las rapaces Falco peregrinus y Chondrohierax uncinatus están consideradas como amenazadas o con poblaciones reducidas en Costa Rica. Finalmente, documentamos un híbrido de Vermivora chrysoptera x $V$. pinus que es raro en el país, aunque no se incluyó como una especie separada (Anexo 1). Precisamente fue $V$. chrysoptera la única especie que se encuentra en la Lista Roja como cercana a la amenaza (NT), pues las demás especies registradas son de preocupación menor (LC).

Los listados comparados con en el análisis de conglomerados se agruparon principalmente según su cercanía geográfica. No obstante la riqueza comparada entre los listados no tuvo una similitud mayor al 60\%, incluso para el caso de la misma localidad en diferentes décadas (Sede Central UCR). El Recinto de Paraíso fue ligeramente más similar a la Sede Central que a la Sede Occidente, aunque comparte alrededor de un $40 \%$ de la riqueza con los sitios más alejados del Occidente del GAM (Figura 5).

\section{Discusión}

Los resultados respecto a la composición de la riqueza de aves en el Recinto de Paraíso coinciden con otros estudios en zonas urbanas de Costa Rica y el Neotrópico. Por ejemplo, las familias más representativas en las áreas urbanas tropicales son las mismas que documentamos, pues los ambientes modificados son importantes para una gran cantidad de especies carnívoras, granívoras e insectívoras (Ramírez, 2010). También es esperado que la mayoría de especies registradas fueran las aves residentes, pues son las que se reproducen en el sitio o inmediaciones, mientras que las aves migratorias latitudinales únicamente usan estos espacios para alimentarse o descansar cuando están de paso o por periodos cortos (Marín, 2005; Rodríguez y Guido 2017).

Algunas especies de todos los sitios comparados presentan alta abundancias y son dominantes en los espacios urbanos y periurbanos de la GAM (e.g. Zonotrichia capensis, Pygochelidon cyanoleuca, Columbina inca, Pitangus sulphuratus, Cantorchilus modestus Psittacara finschil, Elanus leucurus, Quiscalus mexicanus, Turdus grayi, Myiozetetes similis) (Stiles y Skutch, 1989; Biamonte et al., 2011). Aunque, la gran mayoría de aves documentadas en el Recinto son comunes en zonas abiertas y alteradas, el sitio brinda hábitat para especies que tienen cierta dependencia de bosque secundario y humedal: por ejemplo, muchos miembros registrados dentro de familias como Furnaridae, Picidae, Tytiridae y Thraupidae prefieren bosques secundarios y sus bordes (Stiles y Skutch, 1989). Además, las especies de las familias Ciconidae, Anatidae, Ardeidae, Rallidae, Jacanidae, Charadriidae, la golondrina de río (Riparia riparia) y el mosquero de agua (Sayornis nigricans) están asociadas a humedales (Stiles y Skutch, 1989), y difícilmente se ubicarán en zonas urbanas donde estos ecosistemas han sido erradicados. La presencia de especies raras de observar en el país o amenazadas, especialmente de la familia Parulidae (e.g. V. chrysoptera y sus híbrido, Setophaga magnolia, S. striata, S. dominica) (Garrigues y Dean, 2014), indica que Paraíso de Cartago presenta condiciones de hábitat favorables para estas migratorias neárticas.

Probablemente el listado sería solo una pálida muestra de la riqueza de aves que la zona de Paraíso de Cartago hubo de tener en décadas pasadas, pero la riqueza del Recinto de Paraíso no deja de ser considerable. A pesar de poseer menor extensión que las demás áreas muestreadas en los otros esfuerzos analizados, nuestro sitio obtuvo un 57\% de la cantidad de especies registradas históricamente para la Sede Central UCR, y solo un 16\% menos que el último listado publicado para ese campus. Se registra al menos 10\% más especies que las reportadas para la Sede Occidente UCR, y entre un 38 a 50\% más especies que los otros sitios inventariados de San Ramón y San José, respectivamente. Comparando con otras zonas Neotropicales cercanas, El Recinto de Paraíso, a pesar de tener un territorio mucho menor a la Universidad del Quindío y Universidad del Valle, ambos campus a una altura similar a nuestro sitio en Colombia, presentó solo 7\% menos y 22,5 \% más especies, respectivamente (Marín, 2005; Muñoz, Fierro y Rivera, 2007). No podemos subestimar la importancia que tienen los 
campus universitarios como repositorios de aves en la GAM: la riqueza de los campus de Universidad de Costa Rica evaluados equivale aproximadamente el 50\% del número de especies registradas dentro del círculo del Conteo Navideño de Aves de Estación Biológica La Selva y reservas aledañas (535 especies), siendo Sarapiquí una de las áreas más diversas de aves en la región (OET, 2018).

Se concluye con que la ciudad de Paraíso de Cartago posee características biogeográficas y de paisajes favorables para una alta diversidad, y por ende, conservación de las aves: tiene la influencia de las IBAS (Áreas de Importancia para las Aves) de la Cordillera Volcánica Central (Zona de Cartago del Valle Central) y de la Amistad Caribe (Valles de Orosí y Turrialba), siendo ambas IBAS dentro de las más diversas para Costa Rica (Sandoval y Sánchez, 2012). Paraíso de Cartago es los pocos sitios del GAM que todavía conservan lagunas (Acosta-Chaves y Jiménez, 2016): esto reflejado en los humedales del Jardín Botánico Lankester, el Parque recreativo La Expresión (Laguna Doña Ana) y Lagunas Cóncavas, todas cercanas al Recinto de Paraíso. No obstante, la permanencia de estos sistemas, incluyendo la ciénaga del Recinto, peligra por urbanización y procesos de eutrofización, sin permitir sitios donde se puedan formar nuevas lagunas naturalmente (Acosta-Chaves, obs. pers.). La pérdida de humedales es una de las causas de menor diversidad de aves registradas recientemente respecto a décadas pasadas en otros campus como la Sede Central UCR (Biamonte et al., 2011), y son fundamentales para la permanencia de procesos ecológicos que involucran aves de humedal en Paraíso de Cartago (Acosta, 2013; Acosta-Chaves y Jiménez, 2016).

Si bien documentar la biodiversidad del GAM servirá como base de comparación para futuros indicadores sobre los cambios que sufrirá la biodiversidad en la zona, no se puede permanecer en ese punto. A sabiendas que la tasa de extinción de aves del GAM se incrementará con el aumento de la urbanización (Biamonte et al., 2011), ciertamente la existencia de fragmentos de bosque y humedales puede significar una solución primaria a ese problema al permitir las interconexiones entre núcleos boscosos (Krauczuk, 2005). Además, identificar sitios que reúnan la confluencia de varios ecosistemas en áreas pequeñas, como el Recinto de Paraíso, es fundamental para conservar la mayor cantidad de especies, pues no todas las áreas verdes en la matriz urbana mantienen el mismo porcentaje de biodiversidad (Strohbach, Haase y Kabisch, 2009).

Finalmente, es importante considerar que instituciones como la Universidad de Costa Rica deben velar por conservar la rica avifauna que poseen sus fincas. Además de preservar los remanentes de vegetación y humedales que existen, debe realizar otras acciones que favorezcan la convivencia con la biodiversidad contenida en sus campus. Por ejemplo, sería importante distribuir el uso del suelo, reforestar, construir o remodelar siguiendo un diseño verde basado realmente en proveer beneficios a la biodiversidad local y otros objetivos medioambientales (Wells, Böhm, Boch, Fischer y Kalko, 2011), o implementar medidas de mitigación contra estructuras dañinas para las aves como los ventanales (Wells et al., 2011; MenachoOdio, 2018). En un escenario optimista donde los campus se administren bajo esos principios, se prevería que el listado de aves del Recinto de Paraíso se enriqueciera a futuro con especies de bosque secundario y humedal. No obstante, se han removido árboles del bosquecito del Recinto de Paraíso, por lo que es importante sean sustituidos por especies nativas que brinden alimento para la fauna (Ramírez, obs.pers.). Esfuerzos futuros para determinar no solamente la riqueza de aves, sino su abundancia, permitirá un mejor entendimiento de las dinámicas ecológicas y poblacionales de las aves en el campus; así como oportunidades y amenazas para su conservación.

\section{Agradecimientos}

Este esfuerzo es in memoriam de D. Aguilar-García, quien colaboró activamente con la elaboración de este listado. B. Araya, K. Quirós, K. Gómez y S. Hernández contribuyeron con muestreos y observaciones personales que aportaron para enriquecer la investigación. Finalmente, agradecer facilidades brindadas por el Recinto de Paraíso. 


\section{Bibliografía}

Acosta, V. (2013). Pérdida de hábitat y biodiversidad en la ciudad de Heredia, Costa Rica. Ambientico 233, 64-74.

Acosta-Chaves, V. y Jiménez, D. (2016). Feeding behavior of the Least Grebe (Tachybaptus dominicus) upon neotropical ranids in Costa Rica. Florida Field Naturalist, 44, 49-52

Brenes, R. y Brenes, V. (2016). Informe de la calidad del agua del Humedal ubicado en la UCR. Cartago, Costa Rica: Grupo Globe, Liceo de Paraíso

Biamonte, E., Sandoval, L., Chacón, E. y Barrantes, G. (2011). Effect of urbanization on the avifauna in a tropical metropolitan area. Landscape Ecology, 26, 183-194.

Cerdas-Fernández, C. (2014). Evaluación en parques urbanos sobre la avifauna en el sureste del cantón central de San José, Costa Rica. (Tesis de licenciatura). Universidad de Costa Rica. Heredia Costa Rica.

CITES. (2018). Apéndices I, II y III de la CITES. Disponible en: https:speciesplus.net. Consultada el 23 de septiembre del 2018.

Clergeau, P., Isaacson, A., Murton, R. y Falardeau, G. (1998). Bird abundance and diversity along an urban-rural gradient: a comparative study between two cities on different continents. Condor, 100, 413-425.

Escobar-Ibañez, J. y MacGregor-Fors, I. (2017). What's new? An Updated Review of Avian Ecology in Urban Latin America. Pp. 11-31. En MacGregorFors, I. y Escobar-Ibañez, J. (Ed.), Avian Ecology in Latin American Cityscapes. Cham, Suiza: Springer Nature.
Garrigues, R. y Dean, R. (2014). The Birds of Costa Rica a field guide. Segunda Edición. Ithaca, New York: Zona Tropical. 426pp.

Guido, I. y Rodríguez, C. (2009). Avifauna del bosque municipal José Figueres Ferrer, San Ramón, Alajuela, Costa Rica (Noviembre 2006 a Mayo 2009). Zeledonia, 13(1), 20-27.

Hammer, Ø., Harper, D. y Ryan, P. (2001). Past: Palaeontological Statistics software package for education and data analysis. Palaeontología Electronica 4(1), 2-9.

Holdridge, L. (1987). Ecología basada en zonas de vida. San José, Costa Rica: Instituto Iberoamericano de Cooperación para la agricultura. 216 pp.

Krauczuk, E. (2005). Aves do Inta-Campo Anexo ao Zaiman, e do Campus da Universidade Nacional de Misiones, Posadas, Misiones, Argentina. Actualidades Ornitológicas, 126, 17-39.

Leveau, L. y Leveau, C. (2004). Comunidades de aves en un gradiente urbano de la ciudad de Mar del Plata, Argentina. Hornero, 19(1),13-21.

Marín, O. (2005). Avifauna del campus de la Universidad del Quindío. Boletín SAO, 15(02), 42-60.

Menacho-Odio, R. (2018). Colisión de aves con ventanas: problema, prevención, mitigación y tendencias de investigación. Zeledonia, 22(1), 59-76.

MINAE. (2005). Lista de especies de aves con poblaciones reducidas y en Peligro de Extinción para Costa Rica Decreto Ejecutivo 32633-MINAE. La Gaceta, 20 de septiembre del 2005.

Molina, E., Gamboa, J., y González, P. (1991). Fertilización potásica de Gypsophila paniculata cv. perfecta en Paraíso, Cartago. Revista Agronomía costarricense, 15(1/2), 185-186. 
Muñoz, M. C., Fierro-Calderón, K., y Rivera-Gutierrez, H. (2007). Las aves del campus de la Universidad del Valle, una isla verde urbana en Cali, Colombia. Ornitología Colombiana, 5(5), 5-20.

OET. (2018). La Selva Conteo de Aves Navideño. Organización para Estudios Tropicales. Disponible en: https://sura.ots.ac.cr/cbc//conteo2017.php. Consultada el 23 de septiembre del 2018.

Ralph, J., Geupel, G., Pyle, P., Martin, T., De Sante, D. y Milá, B. (1996). Manual de métodos de campo para el monitoreo de aves terrestres. Departamento de Agricultura. California, Estados Unidos. 46 pp.

Ramírez, J. (2010). Diversidad de aves de hábitats naturales y modificados en un paisaje de la Depresión Central de Chiapas, México. Revista de Biología Tropical, 58, 511-528.

Rodríguez-Abarca S., I. Vargas-Valverde. (2016). Estimación de la densidad, abundancia y riqueza de aves en un ecosistema urbano, Campus Omar Dengo, Universidad Nacional, Heredia Costa Rica. Disponible en: http://www.rpubs.com/Isabel_ Vargas/185944. Consultada el 23 de septiembre del 2018.

Rodríguez, C. y Guido, I. (2008). Lista preliminar de la avifauna de la Ciudad Universitaria Carlos Monge Alfaro de la Universidad de Costa Rica, San Ramón 2006-2008. Inter Sedes, 16, 11-22.

Rodríguez, C. y Guido, I. (2017). Diversidad y abundancia de aves de la Reserva Biológica Alberto Manuel Brenes y su área de amortiguamiento, Costa Rica. Revista Pensamiento Actual, 17, 74-95.

Sandoval, L. y Sánchez, C. (2012). Áreas Importantes para la Conservación de las Aves de Costa Rica. San José, Costa Rica: Unión de Ornitólogos de Costa Rica. 196pp.
Sandoval, L. y Sánchez, C (2019). Lista de aves de Costa Rica: vigésima octava actualización. Unión de Ornitólogos de Costa Rica. San José, Costa Rica. Disponible en: http://uniondeornitologos.com/wp-content/ uploads/2019/08/Lista-de-Aves-de-Costa-RicaXXVIII.pdf. Consultada el 28 de septiembre del 2019.

Soto, M. (2016). Ciencia favorece práctica del aviturismo en Costa Rica. Periódico La Nación. Disponible en: https://www.nacion.com/ ciencia/medio-ambiente/ciencia-favorecepractica-del-aviturismo-en-costarica/ CISZV4FNHVH6LAV45XNTTXIZKI/story/. Consultada el 23 de septiembre del 2018.

Stiles, G. (1990). La Avifauna de la Universidad de Costa Rica y sus alrededores a través de veinte años (1968-1989). Revista de Biología Tropical, 38(2B), 361-381.

Stiles, G. y Skutch, A. (1989). A guide to the Birds of Costa Rica. Ithaca, New York, E.U.A: Cornell University Press. 572 pp.

Strohbach, M. W., Haase, D., y Kabisch, N. (2009). Birds and the city: urban biodiversity, land use, and socioeconomics. Ecology and Society, 14(2), 31.

UICN. (2018). Lista Roja de Especies Amenazadas de UICN. Disponible en: http://www.iucnredlist. org/. Consultada el 23 de septiembre del 2018.

Wells, K., Böhm, S., Boch, S., Fischer, M., y Kalko, E. (2011). Local and landscape-scale forest attributes differ in their impact on bird assemblages across years in forest production landscapes. Basic and applied ecology, 12(2), 97-106.

Xeno-canto Foundation. (2018). Xeno-canto Foundation and Naturalist Biodiversity Center. Disponible en: https://www.xeno-canto.org/. Consultada el 23 de septiembre del 2018.

Zamora, N. (2008). Unidades fitogeográficas para la clasificación de ecosistemas terrestres en Costa Rica. Recursos Naturales y Ambiente, 54, 14-20. 


\section{CUADRO 1}

\section{Aves del campus del Recinto de Paraíso (Enero 2016-Enero 2018). Estatus residente (R) o migratorio (M).}

\begin{tabular}{|c|c|c|c|c|c|}
\hline \multicolumn{2}{|r|}{ Especie } & Common Name & Nombre común & Estatus & $\begin{array}{l}\text { Apéndice } \\
\text { CITES }\end{array}$ \\
\hline \multicolumn{6}{|c|}{ Anseriformes } \\
\hline \multicolumn{6}{|c|}{ Anatidae (1) } \\
\hline 1 & $\begin{array}{l}\text { Dendrocygna } \\
\text { autumnalis }\end{array}$ & $\begin{array}{c}\text { Black-bellied } \\
\text { Whistling-Duck }\end{array}$ & Pijije Común & $\mathrm{R}$ & \\
\hline \multicolumn{6}{|c|}{ Galliformes } \\
\hline \multicolumn{6}{|c|}{ Cracidae (1) } \\
\hline 2 & $\begin{array}{c}\text { Ortalis } \\
\text { cineireceps }\end{array}$ & $\begin{array}{l}\text { Gray-headed } \\
\text { Chachalaca }\end{array}$ & Chachalaca Cabecigrís & $\mathrm{R}$ & \\
\hline \multicolumn{6}{|c|}{ Odontophoridae (1) } \\
\hline 3 & $\begin{array}{l}\text { Colinus } \\
\text { cristatus }\end{array}$ & Crested Bobwhite & Codorniz Crestada & $\mathrm{R}$ & \\
\hline \multicolumn{6}{|c|}{ Columbiformes } \\
\hline \multicolumn{6}{|c|}{ Culumbidae (5) } \\
\hline 4 & $\begin{array}{l}\text { Patagioenas } \\
\text { flavirostris }\end{array}$ & Red-billed Pigeon & $\begin{array}{l}\text { Paloma Morada } \\
\text { Común }\end{array}$ & $\mathrm{R}$ & \\
\hline 5 & $\begin{array}{l}\text { Patagioenas } \\
\text { fasciata }\end{array}$ & $\begin{array}{l}\text { Band-tailed } \\
\text { Pigeon }\end{array}$ & Paloma Collareja & $\mathrm{R}$ & \\
\hline 6 & Columbina inca & Inca Dove & Tortolita Colilarga & $\mathrm{R}$ & \\
\hline 7 & $\begin{array}{l}\text { Columbina } \\
\text { talpacoti }\end{array}$ & $\begin{array}{l}\text { Ruddy Ground } \\
\text { Dove }\end{array}$ & Tortolita Rojiza & $\mathrm{R}$ & \\
\hline 8 & Zenaida asiatica & $\begin{array}{l}\text { White-winged } \\
\text { Dove }\end{array}$ & Paloma Aliblanca & $\mathrm{R}, \mathrm{M}$ & \\
\hline \multicolumn{6}{|c|}{ Cuculiformes } \\
\hline \multicolumn{6}{|c|}{ Cuculidae (2) } \\
\hline 9 & Piaya cayana & Squirrel Cuckoo & Cuco Ardilla & $\mathrm{R}$ & \\
\hline 10 & $\begin{array}{l}\text { Crotophaga } \\
\text { sulcirostris }\end{array}$ & Groove-billed Ani & Tijo & $\mathrm{R}$ & \\
\hline \multicolumn{6}{|c|}{ Caprimulgiformes } \\
\hline
\end{tabular}




\begin{tabular}{|c|c|c|c|c|c|}
\hline \multicolumn{6}{|c|}{ Caprimulgidae (1) } \\
\hline 11 & $\begin{array}{l}\text { Nyctidromus } \\
\text { albicollis }\end{array}$ & $\begin{array}{l}\text { Common } \\
\text { Nighthawk }\end{array}$ & Cuyeo & $\mathrm{R}$ & \\
\hline \multicolumn{6}{|c|}{ Apodiformes } \\
\hline \multicolumn{6}{|c|}{ Apodidae (2) } \\
\hline 12 & Chaetura vauxi & Vaux's Swift & Vencejo Común & $\mathrm{R}$ & \\
\hline 13 & $\begin{array}{l}\text { Streptoprocne } \\
\text { zonaris }\end{array}$ & $\begin{array}{l}\text { White-collared } \\
\text { Swift }\end{array}$ & Vencejón Collarejo & $\mathrm{R}$ & \\
\hline \multicolumn{6}{|c|}{ Trochilidae (5) } \\
\hline 14 & Colibri cyanotus & Lesser Violetear & $\begin{array}{c}\text { Colibrí Orejivioláceo } \\
\text { Verde }\end{array}$ & $\mathrm{R}$ & II \\
\hline 15 & $\begin{array}{l}\text { Selasphorus } \\
\text { scintilla }\end{array}$ & $\begin{array}{l}\text { Scintillant } \\
\text { Hummingbird }\end{array}$ & $\begin{array}{l}\text { Colibrí Chispita } \\
\text { Gorginaranja }\end{array}$ & R-END & II \\
\hline 16 & $\begin{array}{l}\text { Chlorostilbon } \\
\text { assimilis }\end{array}$ & Garden Emerald & $\begin{array}{c}\text { Colibrí Esmeralda } \\
\text { Jardinero }\end{array}$ & R-END & II \\
\hline 17 & $\begin{array}{l}\text { Amazilia } \\
\text { hoffmanni }\end{array}$ & $\begin{array}{l}\text { Blue-vented } \\
\text { Hummingbird }\end{array}$ & Amazilia Coliazul & $\mathrm{R}$ & II \\
\hline 18 & Amazilia tzacatl & $\begin{array}{l}\text { Rufous-tailed } \\
\text { Hummnigbird }\end{array}$ & Amazilia Rabirrufa & $\mathrm{R}$ & II \\
\hline 19 & $\begin{array}{l}\text { Hylocharis } \\
\text { eliciae }\end{array}$ & $\begin{array}{l}\text { Blue-throated } \\
\text { Goldentail }\end{array}$ & Colibrí Colidorado & $\mathrm{R}$ & II \\
\hline \multicolumn{6}{|c|}{ Gruiformes } \\
\hline \multicolumn{6}{|c|}{ Rallidae (1) } \\
\hline 20 & $\begin{array}{l}\text { Laterallus } \\
\text { albiguralis }\end{array}$ & $\begin{array}{l}\text { White-throated } \\
\text { Crake }\end{array}$ & Huevo frito & $\mathrm{R}$ & \\
\hline \multicolumn{6}{|c|}{ Charadriidae (2) } \\
\hline 21 & $\begin{array}{l}\text { Vanellus } \\
\text { chilensis }\end{array}$ & $\begin{array}{l}\text { Southern } \\
\text { Lapwing }\end{array}$ & Chorlitazo Sureño & $\mathrm{R}$ & \\
\hline 22 & $\begin{array}{c}\text { Charadrius } \\
\text { vociferus }\end{array}$ & Killdeer & Pijije & $\mathrm{R}, \mathrm{M}$ & \\
\hline \multicolumn{6}{|c|}{ Jacanidae (1) } \\
\hline 23 & Jacana spinosa & Northern Jacana & $\begin{array}{c}\text { Jacana } \\
\text { Centroamericana }\end{array}$ & $\mathrm{R}$ & \\
\hline \multicolumn{6}{|c|}{ Ciconiiformes } \\
\hline \multicolumn{6}{|c|}{ Ciconiidae (1) } \\
\hline 24 & $\begin{array}{l}\text { Mycteria } \\
\text { americana }\end{array}$ & Wood Stork & Cigüeñón & $\mathrm{R}, \mathrm{M}$ & \\
\hline
\end{tabular}




\begin{tabular}{|c|c|c|c|c|c|}
\hline \multicolumn{6}{|c|}{ Pelecaniformes } \\
\hline \multicolumn{6}{|c|}{ Ardeidae (5) } \\
\hline 25 & Ardea alba & Great Egret & Garza Real & $\mathrm{R}, \mathrm{M}$ & \\
\hline 26 & Egretta thula & Snowy Egret & Garceta Nivosa & $\mathrm{R}, \mathrm{M}$ & \\
\hline 27 & Egretta caerulea & Little Blue Heron & Garceta Azul & $\mathrm{R}, \mathrm{M}$ & \\
\hline 28 & Bubulcus ibis & Cattle Egret & Garza de Ganado & $\mathrm{R}, \mathrm{M}$ & \\
\hline 29 & $\begin{array}{l}\text { Butorides } \\
\text { virescens }\end{array}$ & Green Heron & Garcilla Verde & $\mathrm{R}, \mathrm{M}$ & \\
\hline \multicolumn{6}{|c|}{ Cathartiformes } \\
\hline \multicolumn{6}{|c|}{ Cathartidae (2) } \\
\hline 30 & $\begin{array}{l}\text { Coragyps } \\
\text { atratus }\end{array}$ & Black Vulture & Zopilote Negro & $\mathrm{R}$ & \\
\hline 31 & Cathartes aura & Turkey Vulture & Zopilote Cabecirrojo & $\mathrm{R}, \mathrm{M}$ & \\
\hline \multicolumn{6}{|c|}{ Accipitriformes } \\
\hline \multicolumn{6}{|c|}{ Accipitridae (7) } \\
\hline 32 & $\begin{array}{c}\text { Chondrohierax } \\
\text { uncinatus }\end{array}$ & Hook-billed Kite & $\begin{array}{c}\text { Gavilán } \\
\text { Piquiganchudo }\end{array}$ & $\mathrm{R}$ & II \\
\hline 33 & $\begin{array}{l}\text { Elanoides } \\
\text { forficatus }\end{array}$ & $\begin{array}{c}\text { Swallow-tailed } \\
\text { Kite }\end{array}$ & Gavilán Tijereta & $\mathrm{R}$ & II \\
\hline 34 & Elanus leucurus & White-tailed Kite & Gavilán Bailarín & $\mathrm{R}$ & II \\
\hline 35 & $\begin{array}{c}\text { Buteo } \\
\text { platypterus }\end{array}$ & $\begin{array}{c}\text { Broad-winged } \\
\text { Hawk }\end{array}$ & Gavilán Aludo & M & II \\
\hline 36 & $\begin{array}{c}\text { Buteo } \\
\text { brachyurus }\end{array}$ & $\begin{array}{l}\text { Short-tailed } \\
\text { Hawk }\end{array}$ & Gavilán Colicorto & $\mathrm{R}, \mathrm{M}$ & II \\
\hline 37 & Buteo Swainsoni & Swainson's Hawk & Gavilán de Swainson & M & II \\
\hline 38 & $\begin{array}{c}\text { Rupornis } \\
\text { magnirostris }\end{array}$ & Roadside Hawk & Gavilán Chapulinero & $\mathrm{R}$ & II \\
\hline \multicolumn{6}{|c|}{ Strigiformes } \\
\hline \multicolumn{6}{|c|}{ Strigidae (3) } \\
\hline 39 & $\begin{array}{l}\text { Pseudoscops } \\
\text { clamator }\end{array}$ & Striped Owl & Búho Listado & $\mathrm{R}$ & II \\
\hline 40 & $\begin{array}{l}\text { Megascops } \\
\text { choliba }\end{array}$ & $\begin{array}{c}\text { Tropical Screech- } \\
\text { Owl }\end{array}$ & Estucurú o Sorococa & $\mathrm{R}$ & II \\
\hline 41 & $\begin{array}{l}\text { Glaucidium } \\
\text { brasilianum }\end{array}$ & $\begin{array}{l}\text { Ferruginous } \\
\text { Pygmy-Owl }\end{array}$ & Majafierro & $\mathrm{R}$ & II \\
\hline \multicolumn{5}{|c|}{ Coraciiformes } & \\
\hline
\end{tabular}




\begin{tabular}{|c|c|c|c|c|c|}
\hline \multirow[b]{2}{*}{42} & \multicolumn{4}{|c|}{ Momotidae (1) } & \\
\hline & $\begin{array}{c}\text { Momotus } \\
\text { lessonii }\end{array}$ & Lesson's Motmot & Pájaro Bobo & $\mathrm{R}$ & \\
\hline \multicolumn{6}{|c|}{ Piciformes } \\
\hline \multicolumn{6}{|c|}{ Ramphastidae (1) } \\
\hline 43 & $\begin{array}{l}\text { Pteroglossus } \\
\text { torquatus }\end{array}$ & Collared Aracari & Cusingo & $\mathrm{R}$ & \\
\hline \multicolumn{6}{|c|}{ Picidae (2) } \\
\hline 44 & $\begin{array}{l}\text { Melanerpes } \\
\text { hoffmannii }\end{array}$ & $\begin{array}{l}\text { Hoffmann's } \\
\text { Woodpecker }\end{array}$ & $\begin{array}{l}\text { Carpintero de } \\
\text { Hoffmann }\end{array}$ & R-END & \\
\hline 45 & $\begin{array}{l}\text { Colaptes } \\
\text { rubiginosus }\end{array}$ & $\begin{array}{l}\text { Golden-olive } \\
\text { Woodpecker }\end{array}$ & $\begin{array}{c}\text { Carpintero Verde } \\
\text { Dorado }\end{array}$ & $\mathrm{R}$ & \\
\hline \multicolumn{6}{|c|}{ Falconiformes } \\
\hline \multicolumn{6}{|c|}{ Falconidae (3) } \\
\hline 46 & $\begin{array}{l}\text { Caracara } \\
\text { cheriway }\end{array}$ & Crested Caracara & Quebrantahuesos & $\mathrm{R}$ & II \\
\hline 47 & $\begin{array}{c}\text { Milvago } \\
\text { chimachima }\end{array}$ & $\begin{array}{l}\text { Yellow-headed } \\
\text { Caracara }\end{array}$ & $\begin{array}{c}\text { Caracara } \\
\text { Cabecigualdo }\end{array}$ & $\mathrm{R}$ & II \\
\hline 48 & Falco peregrinus & Peregrine Falcon & Halcón Peregrino & M & I \\
\hline \multicolumn{6}{|c|}{ Psittaciformes } \\
\hline \multicolumn{6}{|c|}{ Psittacidae (2) } \\
\hline 49 & $\begin{array}{c}\text { Psittacara } \\
\text { finschi }\end{array}$ & $\begin{array}{c}\text { Crimson-fronted } \\
\text { Parakeet }\end{array}$ & Perico Frentirrojo & R-END & II \\
\hline 50 & Pionus senilis & $\begin{array}{c}\text { White-crowned } \\
\text { Parrot }\end{array}$ & Chucuyo & $\mathrm{R}$ & II \\
\hline \multicolumn{6}{|c|}{ Passeriformes } \\
\hline \multicolumn{6}{|c|}{ Furnariidae (2) } \\
\hline 51 & $\begin{array}{c}\text { Lepidocolaptes } \\
\text { souleyetii }\end{array}$ & $\begin{array}{l}\text { Streak-headed } \\
\text { Woodcreeper }\end{array}$ & $\begin{array}{c}\text { Trepador } \\
\text { Cabecirrayado }\end{array}$ & $\mathrm{R}$ & \\
\hline 52 & $\begin{array}{l}\text { Synallaxis } \\
\text { brachyura }\end{array}$ & Slaty Spinetail & Arquitecto Plomizo & $\mathrm{R}$ & \\
\hline \multicolumn{6}{|c|}{ Tyrannidae (13) } \\
\hline 53 & $\begin{array}{c}\text { Elaenia } \\
\text { flavogaster }\end{array}$ & $\begin{array}{l}\text { Yellow-billed } \\
\text { Elaenia }\end{array}$ & Elainia Copetona & $\mathrm{R}$ & \\
\hline 54 & $\begin{array}{c}\text { Elaenia } \\
\text { chiriquensis }\end{array}$ & Lesser Elaenia & Elainia Sabanera & $\mathrm{R}$ & \\
\hline 55 & $\begin{array}{l}\text { Zimmerius } \\
\text { parvus }\end{array}$ & $\begin{array}{l}\text { Mistletoe } \\
\text { Tyrannulet }\end{array}$ & Mosquerito Cejigrís & $\mathrm{R}$ & \\
\hline
\end{tabular}




\begin{tabular}{|c|c|c|c|c|}
\hline 56 & $\begin{array}{l}\text { Todirostrum } \\
\text { cinereum }\end{array}$ & $\begin{array}{l}\text { Common Tody } \\
\text { Flycatcher }\end{array}$ & Espatulilla Común & $\mathrm{R}$ \\
\hline 57 & Contopus virens & $\begin{array}{l}\text { Eastern Wood- } \\
\text { Pewee }\end{array}$ & Pibí Oriental & M \\
\hline 58 & $\begin{array}{l}\text { Contopus } \\
\text { cinereus }\end{array}$ & Tropical Pewee & Pibí Tropical & $\mathrm{R}$ \\
\hline 59 & $\begin{array}{l}\text { Sayornis } \\
\text { nigricans }\end{array}$ & Black Phoebe & Mosquero de Agua & $\mathrm{R}$ \\
\hline 60 & Attila spadiceus & $\begin{array}{l}\text { Bright-rumped } \\
\text { Attila }\end{array}$ & Attila Lomiamarilla & $\mathrm{R}$ \\
\hline 61 & $\begin{array}{l}\text { Myiarchus } \\
\text { tuberculifer }\end{array}$ & $\begin{array}{l}\text { Dusky-capped } \\
\text { Flycatcher }\end{array}$ & Copetón Crestioscuro & $\mathrm{R}$ \\
\hline 62 & $\begin{array}{l}\text { Pitangus } \\
\text { sulphuratus }\end{array}$ & Great Kiskadee & Cristo Fue & $\mathrm{R}$ \\
\hline 63 & $\begin{array}{c}\text { Megarynchus } \\
\text { pitangua }\end{array}$ & $\begin{array}{l}\text { Boat-billed } \\
\text { Flycatcher }\end{array}$ & Mosquerón Picudo & $\mathrm{R}$ \\
\hline 64 & $\begin{array}{l}\text { Myozetetes } \\
\text { similis }\end{array}$ & Social Flycatcher & Mosquero Cejiblanco & $\mathrm{R}$ \\
\hline 65 & $\begin{array}{c}\text { Tyrannus } \\
\text { melancholicus }\end{array}$ & Tropical Kingbird & Tirano Tropical & $\mathrm{R}$ \\
\hline \multicolumn{5}{|c|}{ Tityridae (1) } \\
\hline 66 & $\begin{array}{c}\text { Tityra } \\
\text { semifasciata }\end{array}$ & Masked Tityra & Pájaro Chancho & $\mathrm{R}$ \\
\hline \multicolumn{5}{|c|}{ Vireonidae (4) } \\
\hline 67 & Vireo flavifrons & $\begin{array}{l}\text { Yellow-throated } \\
\text { Vireo }\end{array}$ & Vireo Pechiamarillo & M \\
\hline 68 & $\begin{array}{c}\text { Vireo } \\
\text { philadelphicus }\end{array}$ & $\begin{array}{l}\text { Philadelphia } \\
\text { Vireo }\end{array}$ & Vireo Amarrillento & M \\
\hline 69 & Vireo Olivaceus & Red-eyed Vireo & Vireo Ojirrojo & M \\
\hline 70 & $\begin{array}{c}\text { Vireo } \\
\text { flavoviridis }\end{array}$ & $\begin{array}{c}\text { Yellow-green } \\
\text { Vireo }\end{array}$ & Vireo Cabecigrís & M \\
\hline \multicolumn{5}{|c|}{ Corvidae (1) } \\
\hline 71 & $\begin{array}{l}\text { Psilorhinus } \\
\text { morio }\end{array}$ & Brown Jay & Piapia & $\mathrm{R}$ \\
\hline \multicolumn{5}{|c|}{ Hirundinidae (5) } \\
\hline 72 & $\begin{array}{c}\text { Pygochelidon } \\
\text { cyanoleuca }\end{array}$ & $\begin{array}{l}\text { Blue-and-white } \\
\text { Swallow }\end{array}$ & $\begin{array}{l}\text { Golondrina Azul y } \\
\text { Blanco }\end{array}$ & $\mathrm{R}, \mathrm{M}$ \\
\hline 73 & $\begin{array}{l}\text { Stelgidopteryx } \\
\text { serripennis }\end{array}$ & $\begin{array}{l}\text { Northern Rough- } \\
\text { winged Swallow }\end{array}$ & $\begin{array}{c}\text { Golondrina } \\
\text { Alirrasposa Norteña }\end{array}$ & $\mathrm{R}, \mathrm{M}$ \\
\hline
\end{tabular}




\begin{tabular}{|c|c|c|c|c|}
\hline 74 & $\begin{array}{l}\text { Stelgidopteryx } \\
\text { ruficolis }\end{array}$ & $\begin{array}{l}\text { Southern Rough- } \\
\text { winged Swallow }\end{array}$ & $\begin{array}{c}\text { Golondrina } \\
\text { Alirrasposa Sureña }\end{array}$ & $\mathrm{R}$ \\
\hline 75 & Riparia riparia & Bank Swallow & Golondrina Ribereña & M \\
\hline 76 & Hirundo rustica & Barn Swallow & Golondrina Tijereta & M \\
\hline \multicolumn{5}{|c|}{ Troglodytidae (2) } \\
\hline 77 & $\begin{array}{l}\text { Troglodytes } \\
\text { aedon }\end{array}$ & House Wren & Soterrey Cucarachero & $\mathrm{R}$ \\
\hline 78 & $\begin{array}{c}\text { Cantorchilus } \\
\text { modestus }\end{array}$ & Cabanis's Wren & Soterrey Chinchirigüí & $\mathrm{R}$ \\
\hline \multicolumn{5}{|c|}{ Turdidae (3) } \\
\hline 79 & $\begin{array}{l}\text { Catharus } \\
\text { fuscater }\end{array}$ & $\begin{array}{c}\text { Slaty-backed } \\
\text { Nightingale- } \\
\text { Thrush }\end{array}$ & Zorzal sombrío & $\mathrm{R}$ \\
\hline 80 & $\begin{array}{l}\text { Catharus } \\
\text { ustulatus }\end{array}$ & $\begin{array}{c}\text { Swainson's } \\
\text { Thrush }\end{array}$ & Zorzal de Swainson & M \\
\hline 81 & Turdus grayi & $\begin{array}{l}\text { Clay-colored } \\
\text { Thrush }\end{array}$ & Yigüirro & $\mathrm{R}$ \\
\hline \multicolumn{5}{|c|}{ Mimidae (1) } \\
\hline 82 & Mimus gilvus & $\begin{array}{c}\text { Tropical } \\
\text { Mockingbird }\end{array}$ & $\begin{array}{c}\text { Pájaro-imitador } \\
\text { Tropical }\end{array}$ & $\mathrm{R}$ \\
\hline \multicolumn{5}{|c|}{ Passeridae (1) } \\
\hline 83 & $\begin{array}{c}\text { Passer } \\
\text { domesticus }\end{array}$ & House Sparrow & Gorrión Común & $\mathrm{R}$ \\
\hline \multicolumn{5}{|c|}{ Fringillidae (2) } \\
\hline 84 & $\begin{array}{c}\text { Euphonia } \\
\text { hirundinacea }\end{array}$ & $\begin{array}{l}\text { Yellow-throated } \\
\text { Euphonia }\end{array}$ & Agüío & $\mathrm{R}$ \\
\hline 85 & Spinus psaltria & Lesser Goldfinch & Mozotillo de Charral & $\mathrm{R}$ \\
\hline \multicolumn{5}{|c|}{ Parulidae (13) } \\
\hline 86 & Mniotila varia & $\begin{array}{c}\text { Black-and-white } \\
\text { Warbler }\end{array}$ & Zebrita & M \\
\hline 87 & $\begin{array}{l}\text { Protonotaria } \\
\text { citrea }\end{array}$ & $\begin{array}{l}\text { Protonotary } \\
\text { Warbler }\end{array}$ & Reinita Cabecidorada & M \\
\hline 88 & $\begin{array}{l}\text { Leiothlypis } \\
\text { peregrina }\end{array}$ & $\begin{array}{l}\text { Tennessee } \\
\text { Warbler }\end{array}$ & Reinita Verdilla & M \\
\hline 89 & $\begin{array}{l}\text { Setophaga } \\
\text { pitiayumi }\end{array}$ & Tropical Parula & Parula Tropical & $\mathrm{R}$ \\
\hline 90 & $\begin{array}{l}\text { Setophaga } \\
\text { magnolia }\end{array}$ & $\begin{array}{l}\text { Magnolia } \\
\text { Warbler }\end{array}$ & Reinita Colifajeada & M \\
\hline
\end{tabular}




\begin{tabular}{|c|c|c|c|c|}
\hline 91 & $\begin{array}{l}\text { Setophaga } \\
\text { castanea }\end{array}$ & $\begin{array}{c}\text { Bay-breasted } \\
\text { Warbler }\end{array}$ & Reinita castaña & M \\
\hline 92 & $\begin{array}{l}\text { Setophaga } \\
\text { striata }\end{array}$ & Blackpoll Warbler & Reinita Rayada & M \\
\hline 93 & Setophaga fusca & $\begin{array}{c}\text { Blackburnian } \\
\text { Warbler }\end{array}$ & Reinita Gorginaranja & M \\
\hline 94 & $\begin{array}{l}\text { Setophaga } \\
\text { petechia }\end{array}$ & Yellow Warbler & Reinita Amarilla & M \\
\hline 95 & $\begin{array}{c}\text { Setophaga } \\
\text { pensylvanica }\end{array}$ & $\begin{array}{l}\text { Chestnut-sided } \\
\text { Warbler }\end{array}$ & $\begin{array}{c}\text { Reinita de Costillas } \\
\text { Castañas }\end{array}$ & M \\
\hline 96 & $\begin{array}{l}\text { Setophaga } \\
\text { dominica }\end{array}$ & $\begin{array}{c}\text { Yellow-throated } \\
\text { Warbler }\end{array}$ & Reinita Gorgiamarilla & $\mathrm{M}$ \\
\hline 97 & $\begin{array}{l}\text { Setophaga } \\
\text { virens }\end{array}$ & $\begin{array}{l}\text { Black-throated } \\
\text { Green Warbler }\end{array}$ & Reinita Cariamarilla & $\mathrm{M}$ \\
\hline 98 & $\begin{array}{l}\text { Basileuterus } \\
\text { rufifrons }\end{array}$ & $\begin{array}{c}\text { Rufous-capped } \\
\text { Warbler }\end{array}$ & Reinita Cabecicastaña & $\mathrm{R}$ \\
\hline \multicolumn{5}{|c|}{ Thraupidae (11) } \\
\hline 99 & $\begin{array}{l}\text { Thraupis } \\
\text { episcopus }\end{array}$ & $\begin{array}{l}\text { Blue-gray } \\
\text { Tanager }\end{array}$ & Viudita & $\mathrm{R}$ \\
\hline 100 & $\begin{array}{l}\text { Thraupis } \\
\text { palmarum }\end{array}$ & Palm Tanager & Tangara Palmera & $\mathrm{R}$ \\
\hline 101 & Stilpnia larvata & $\begin{array}{l}\text { Golden-hooded } \\
\text { Tanager }\end{array}$ & Siete Colores & $\mathrm{R}$ \\
\hline 102 & $\begin{array}{l}\text { Volatinia } \\
\text { jacarina }\end{array}$ & $\begin{array}{c}\text { Blue-black } \\
\text { Grassquit }\end{array}$ & Pius & $\mathrm{R}$ \\
\hline 103 & $\begin{array}{c}\text { Ramphocelus } \\
\text { passerinii }\end{array}$ & $\begin{array}{l}\text { Scarlet-rumped } \\
\text { Tanager }\end{array}$ & Sargento & $\mathrm{R}$ \\
\hline 104 & Coereba flaveola & Bananaquit & Reinita Mielera & $\mathrm{R}$ \\
\hline 105 & Tiaris olivaceus & $\begin{array}{l}\text { Yellow-faced } \\
\text { Grassquit }\end{array}$ & Gallito & $\mathrm{R}$ \\
\hline 106 & $\begin{array}{l}\text { Sporophila } \\
\text { corvina }\end{array}$ & $\begin{array}{l}\text { Variable } \\
\text { Seedeater }\end{array}$ & Setillero Collarejo & $\mathrm{R}$ \\
\hline 107 & $\begin{array}{l}\text { Saltator } \\
\text { maximus }\end{array}$ & $\begin{array}{l}\text { Buff-throated } \\
\text { Saltator }\end{array}$ & $\begin{array}{l}\text { Chayotero, } \\
\text { Comepuntas }\end{array}$ & $\mathrm{R}$ \\
\hline 108 & Saltator atriceps & $\begin{array}{l}\text { Black-headed } \\
\text { Saltator }\end{array}$ & $\begin{array}{l}\text { Chayotero, } \\
\text { Comepuntas }\end{array}$ & $\mathrm{R}$ \\
\hline 109 & $\begin{array}{c}\text { Saltator } \\
\text { coerulescens }\end{array}$ & Grayish Saltator & Saltator Grisáceo & $\mathrm{R}$ \\
\hline \multicolumn{5}{|c|}{ Emberizidae (2) } \\
\hline 110 & $\begin{array}{c}\text { Melozone } \\
\text { leucotis }\end{array}$ & $\begin{array}{c}\text { White-eared } \\
\text { Ground-Sparrow }\end{array}$ & Cuatro Ojos & $\mathrm{R}$ \\
\hline
\end{tabular}




\begin{tabular}{|c|c|c|c|c|}
\hline 111 & $\begin{array}{l}\text { Zonotrichia } \\
\text { capensis }\end{array}$ & $\begin{array}{l}\text { Rufous-collared } \\
\text { Sparrow }\end{array}$ & Come maíz, Pirrís & $\mathrm{R}$ \\
\hline \multicolumn{5}{|c|}{ Cardinalidae (3) } \\
\hline 112 & Piranga rubra & Summer Tanager & Tangara Veranera & M \\
\hline 113 & Piranga olivacea & Scarlet Tanager & Tangara Escarlata & M \\
\hline 114 & $\begin{array}{c}\text { Piranga } \\
\text { bidentata }\end{array}$ & $\begin{array}{c}\text { Flame-colored } \\
\text { Tanager }\end{array}$ & Tangara Dorsirrayada & $\mathrm{R}$ \\
\hline \multicolumn{5}{|c|}{ Icteridae (6) } \\
\hline 115 & Sturnella magna & $\begin{array}{c}\text { Eastern } \\
\text { Meadowlark }\end{array}$ & Zacatera & $\mathrm{R}$ \\
\hline 116 & Dives Dives & $\begin{array}{l}\text { Melodius } \\
\text { Blackbird }\end{array}$ & Tordo Cantor & $\mathrm{R}$ \\
\hline 117 & $\begin{array}{l}\text { Quiscalus } \\
\text { mexicanus }\end{array}$ & $\begin{array}{l}\text { Great-tailed } \\
\text { Grackle }\end{array}$ & Zanate Grande & $\mathrm{R}$ \\
\hline 118 & $\begin{array}{l}\text { Molothrus } \\
\text { oryzovorus }\end{array}$ & Giant Cowbird & Vaquero Grande & $\mathrm{R}$ \\
\hline 119 & Icterus galbula & Baltimore Oriole & Cacique Veranero & M \\
\hline 120 & $\begin{array}{l}\text { Psarocollus } \\
\text { montezuma }\end{array}$ & $\begin{array}{l}\text { Montezuma } \\
\text { Oropendola }\end{array}$ & Oropéndola & $\mathrm{R}$ \\
\hline $\mathrm{X}$ & $\begin{array}{l}\text { V.chrysoptera x } \\
\text { V. pinus }\end{array}$ & \multicolumn{2}{|c|}{ Brewster's Warbler (híbrido) } & M \\
\hline
\end{tabular}




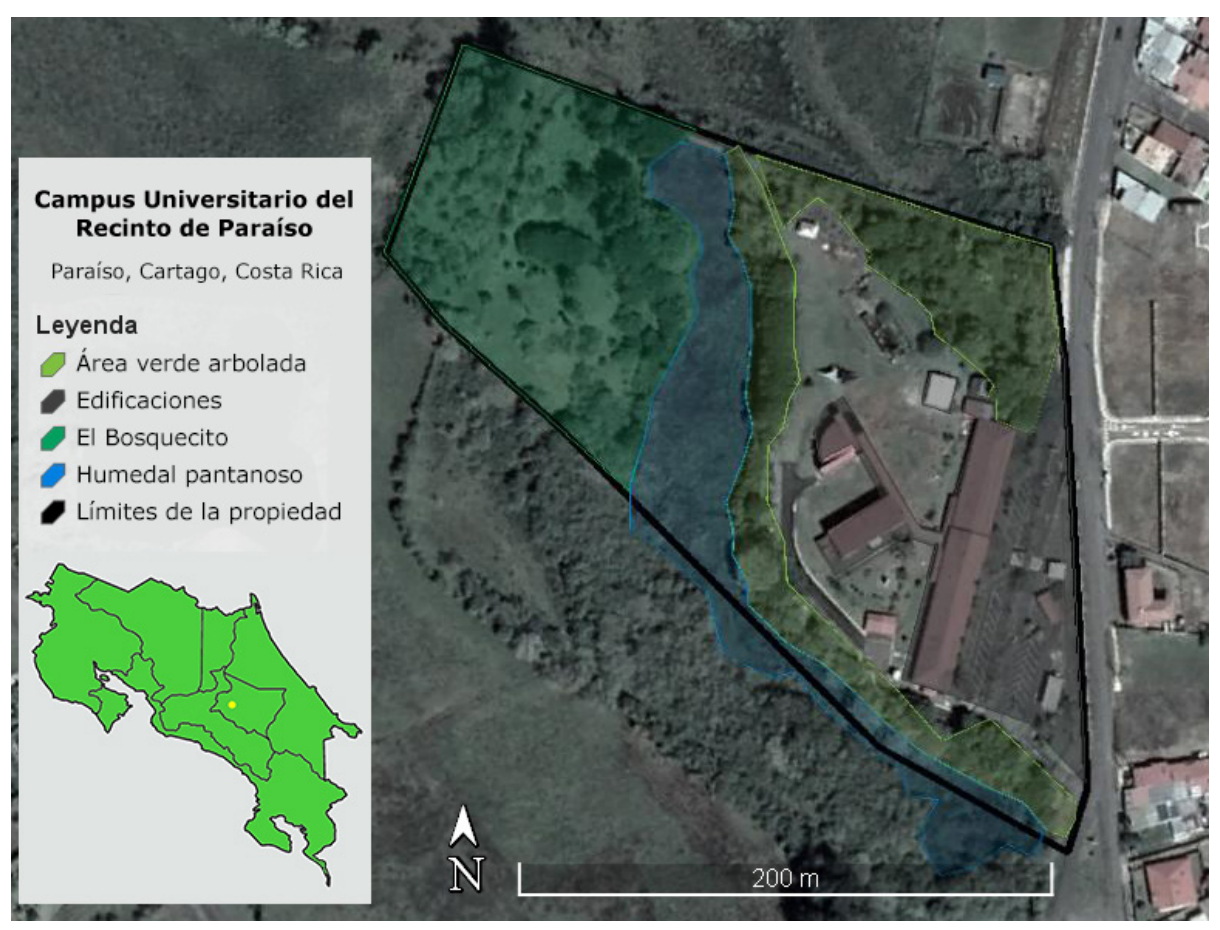

Figura 1. Diagrama del Campus Universitario Rafael Ángel Calderón Guardia (Recinto de Paraíso) de la Universidad de Costa Rica. Elaborado con Google Earth Pro y Quantum GIS.

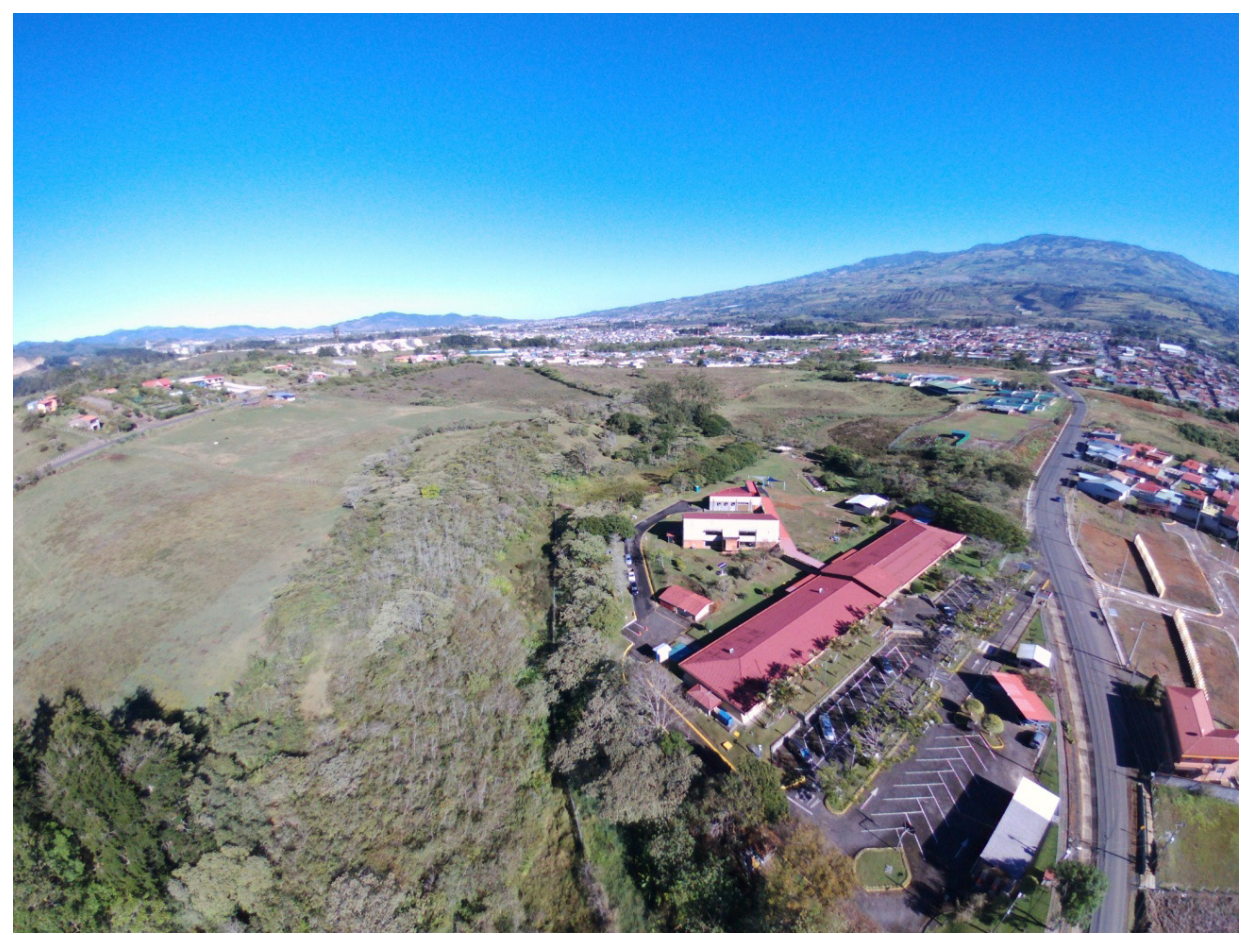

Figura 2. Vista aérea del Campus Universitario Rafael Ángel Calderón Guardia (Recinto de Paraíso) de la Universidad de Costa Rica, Provincia de Cartago, Costa Rica. Al fondo se aprecian el pueblo de Paraíso y el Volcán Irazú. Las instalaciones del campus están rodeadas por un humedal, bosque secundario y pastizales. Foto cortesía de Antonio Loaiza. 


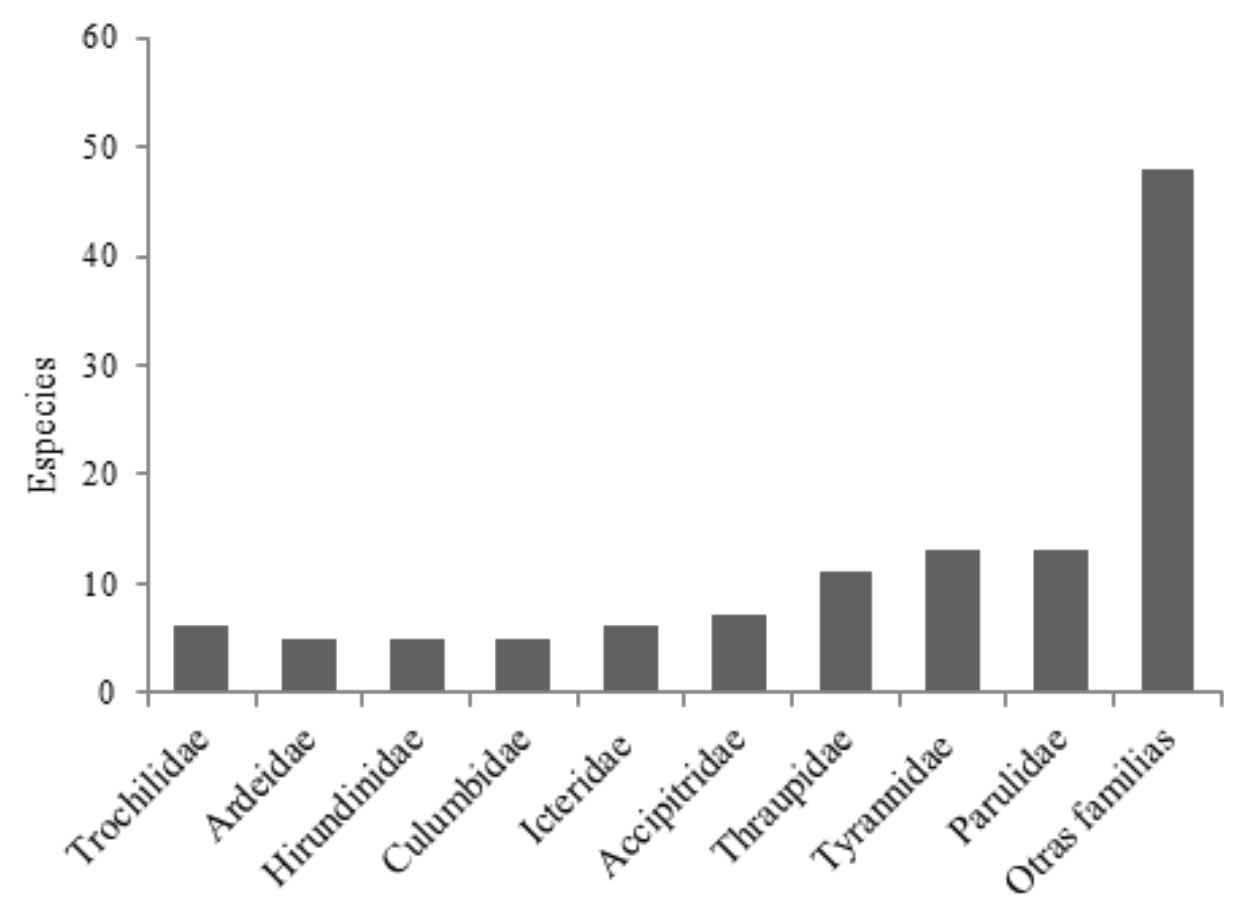

Figura 3. Distribución porcentual de las aves por familia taxonómica encontradas en el Campus Recinto de Paraíso, Costa Rica.

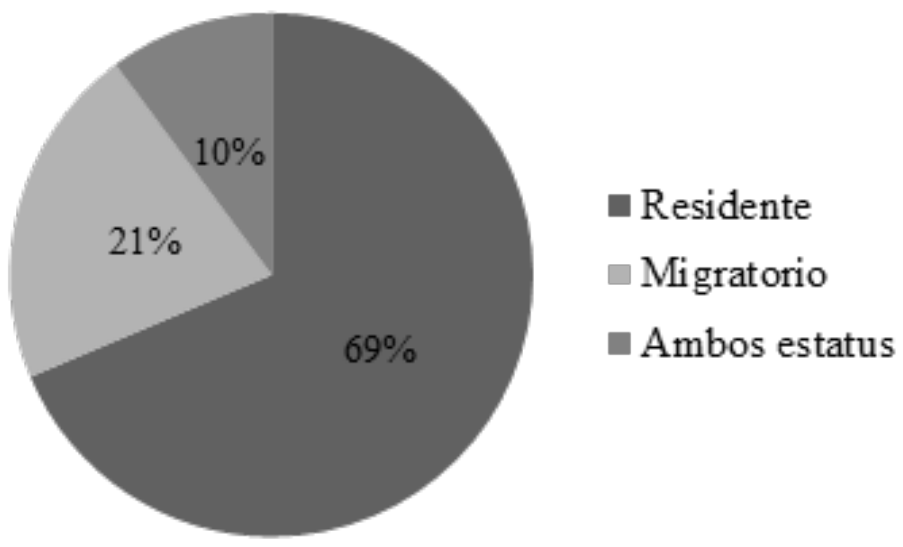

Figura 4. Distribución porcentual de las aves por estatus encontradas en el Campus Recinto de Paraíso, Costa Rica. 


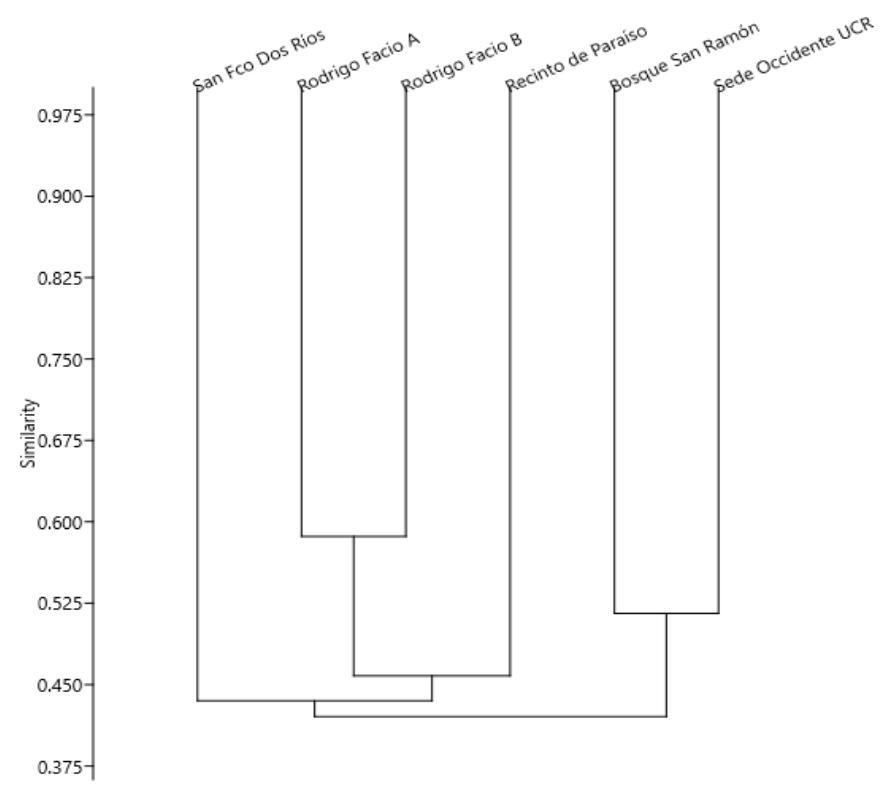

Figura 5. Análisis de conglomerados (vinculación simple, índice de Jaccard) mostrando el grado de similitud entre los listados del Recinto de Paraíso (Cartago, 2016-2018), San Francisco de Dos Ríos (San José, 2010), la Sede Central UCR A (San José, 1968-1989), la Sede Central UCR B (San José, 2004-2009), la Sede Occidente UCR (San Ramón, 2006-2008) y el Bosque Municipal de San Ramón (San Ramón, 2006-2009).
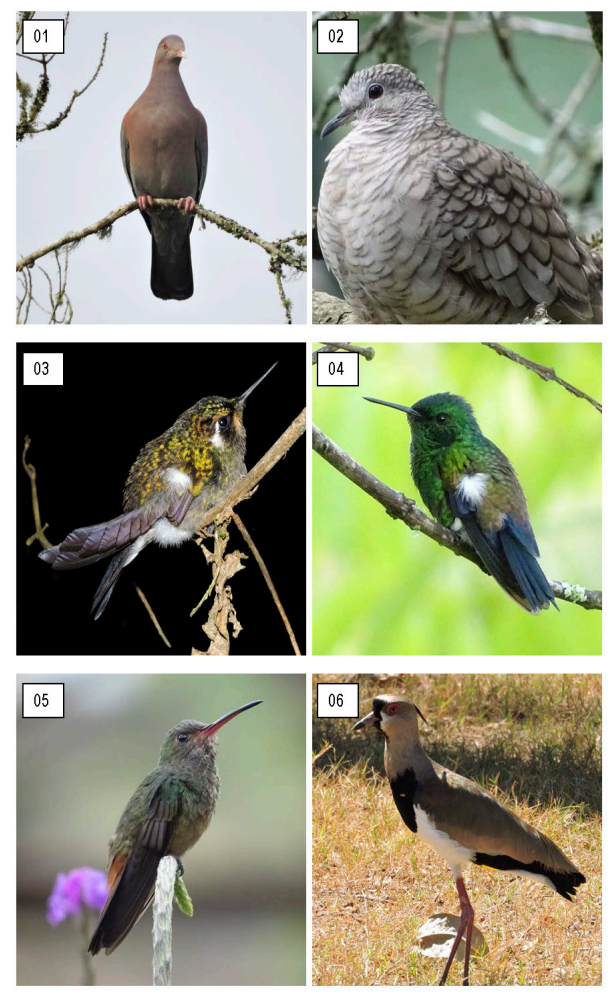

Anexo 1. Algunas aves detectadas en el Recinto de Paraíso. Patagioenas flavirostris (o1), Columbina inca (02), Chlorostilbon assimilis (o3), Amazilia hoffmanni (04), Amazilia tzacatl (05), Vanellus chilensis (06). Fotos por D. Ramírez y V. Acosta. Continúa... 

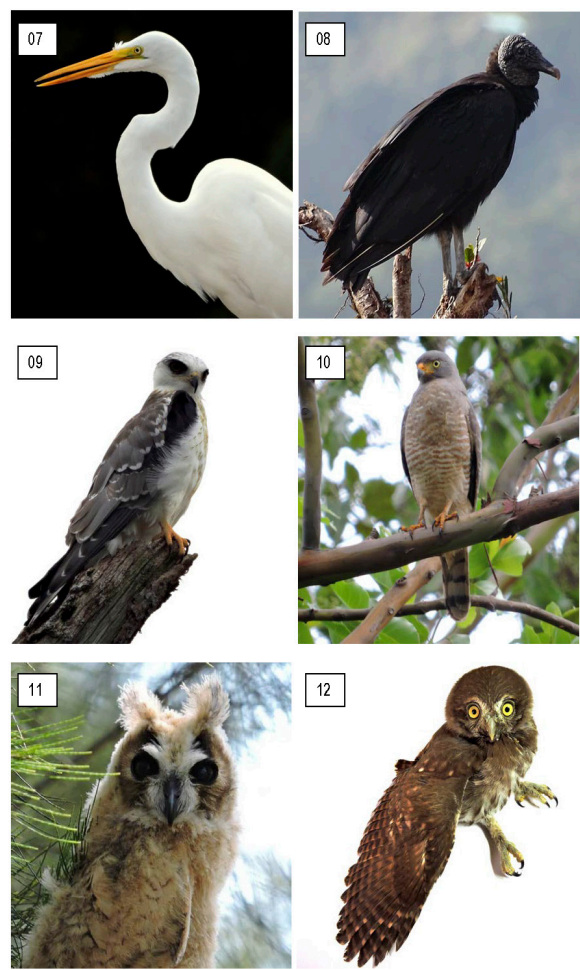

Anexo 1. Continuación. Algunas aves detectadas en el Recinto de Paraíso. Ardea alba (07), Coragyps atratus (o8), Elanus leucurus (og), Rupornis magnirostris (10), Pseudoscops clamator (11), Glaucidium brasilianum (12). Fotos por D. Ramírez y V. Acosta. Continúa...
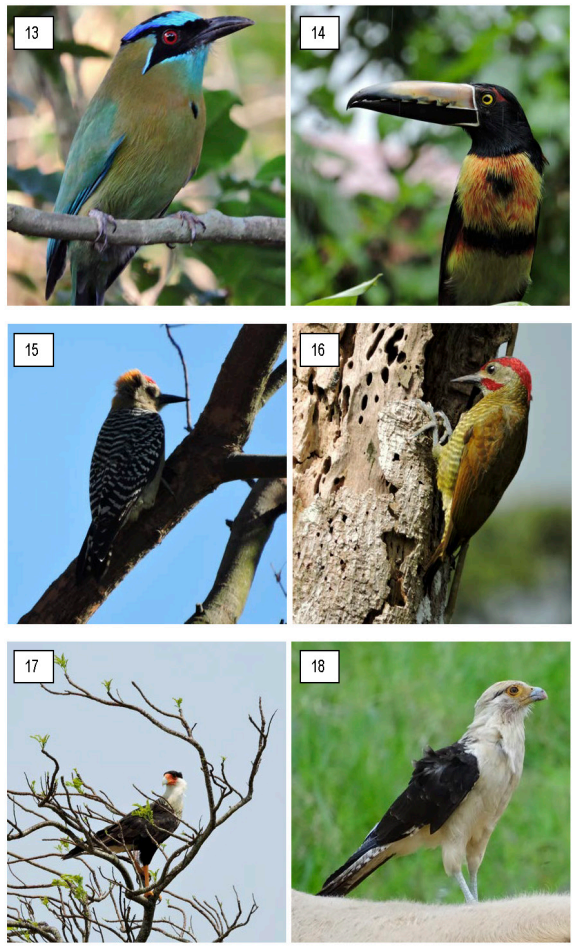

Anexo 1. Continuación. Algunas aves detectadas en el Recinto de Paraíso. Momotus lessonii (13), Pteroglossus torquatus (14), Melanerpes hoffmannii (15), Colaptes rubiginosus (16), Caracara cheriway (17), Milvago chimachima (18). Fotos por D. Ramírez y V. Acosta. Continúa... 

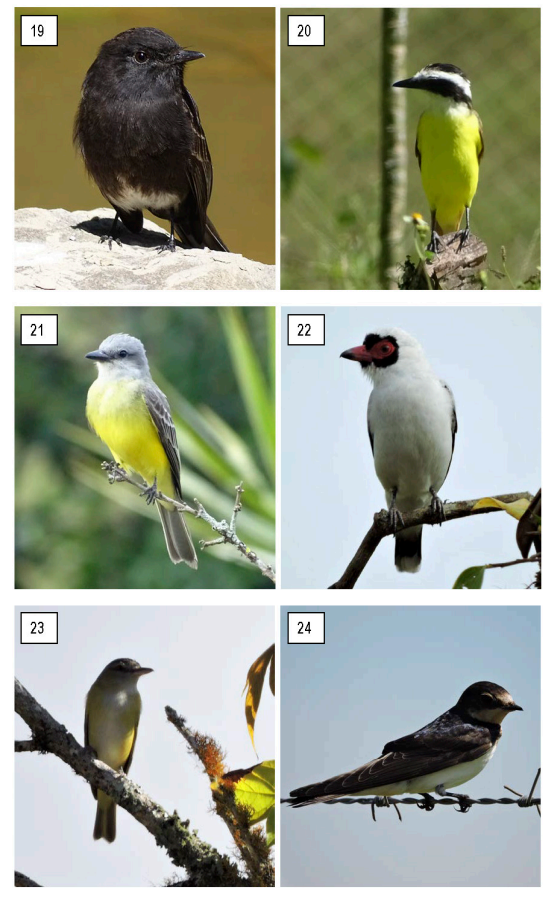

Anexo 1. Continuación. Algunas aves detectadas en el Recinto de Paraíso. Sayornis nigricans (19), Pitangus sulphuratus (20), Tyrannus melancholicus (21),Tityra semifasciata (22), Vireo philladelphicus (23), Hirundo rustica (24). Fotos por D. Ramírez y V. Acosta. Continúa...
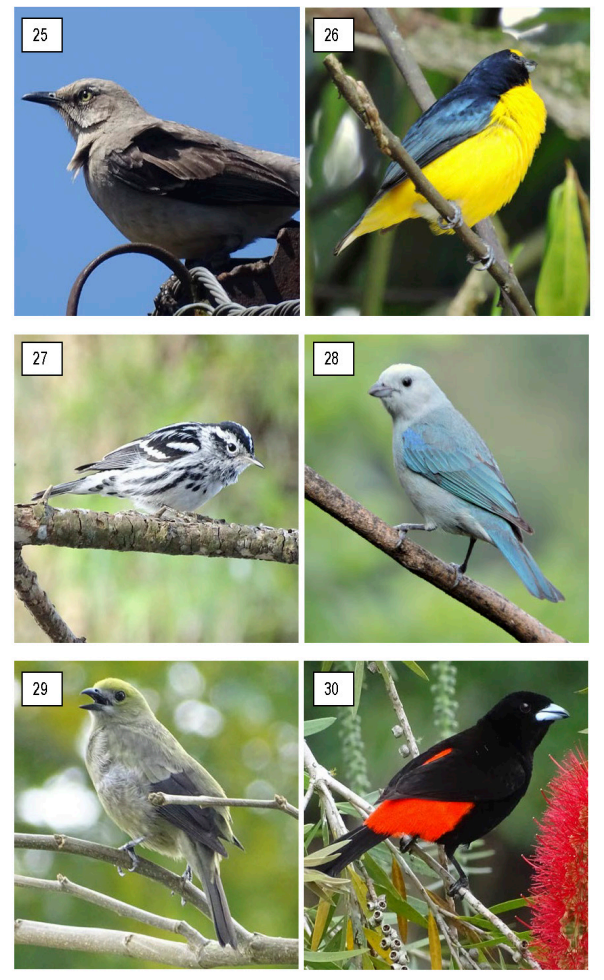

Anexo 1. Continuación. Algunas aves detectadas en el Recinto de Paraíso. Minus gilvus (25), Euphonia hirundinacea (26), Mniotilta varia (27),Thraupis episcopus (28), Thraupis palmarum (29), Ramphocelus passerinii (30). Fotos por D. Ramírez y V. Acosta. Continúa... 

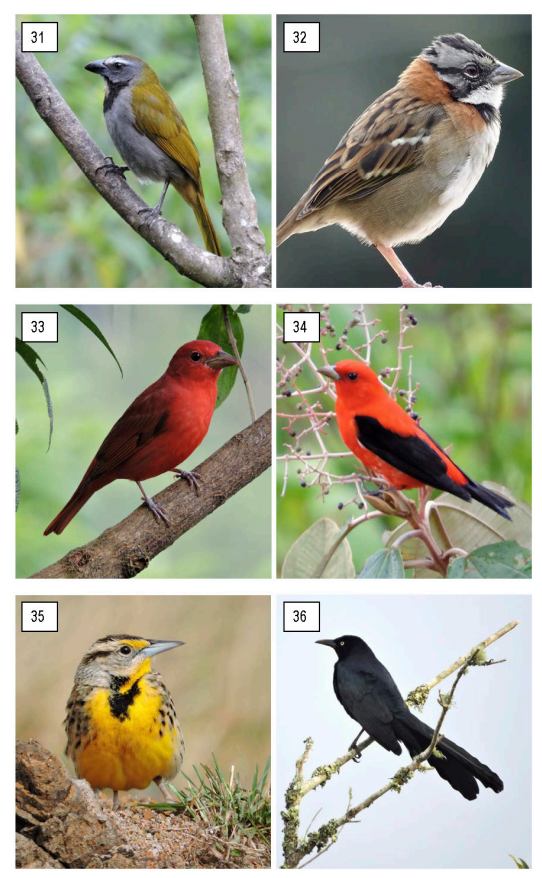

Anexo 1. Continuación. Algunas aves detectadas en el Recinto de Paraíso. Saltator maximus (31), Zonotrichia capensis (32), Piranga rubra (33), Piranga olivacea (34), Sturnella magna (29), Quiscalus mexicanus (30). Fotos por D. Ramírez y V. Acosta. 